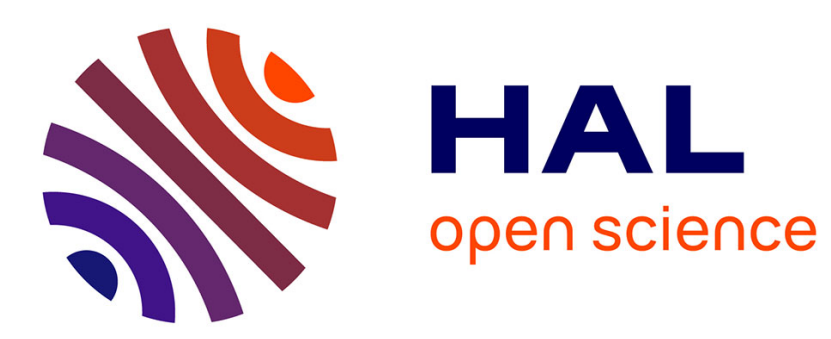

\title{
Observability of Discrete-Time Linear Systems with Communication Protocols and Dropouts
}

\author{
Aneel Tanwani, Raphael Jungers, Maurice Heemels
}

\section{To cite this version:}

Aneel Tanwani, Raphael Jungers, Maurice Heemels. Observability of Discrete-Time Linear Systems with Communication Protocols and Dropouts. 57th IEEE Conference on Decision and Control (CDC 2018), Dec 2018, Miami Beach, FL, United States. 10.1109/CDC.2018.8619308 . hal-01951815

\author{
HAL Id: hal-01951815 \\ https://hal.laas.fr/hal-01951815
}

Submitted on 11 Dec 2018

HAL is a multi-disciplinary open access archive for the deposit and dissemination of scientific research documents, whether they are published or not. The documents may come from teaching and research institutions in France or abroad, or from public or private research centers.
L'archive ouverte pluridisciplinaire HAL, est destinée au dépôt et à la diffusion de documents scientifiques de niveau recherche, publiés ou non, émanant des établissements d'enseignement et de recherche français ou étrangers, des laboratoires publics ou privés. 


\section{Observability of Discrete-Time Linear Systems with Communication Protocols and Dropouts}

\author{
Aneel Tanwani
}

Raphael Jungers

\author{
W.P.M.H. (Maurice) Heemels
}

\begin{abstract}
We consider the problem of analyzing observability in discrete-time linear systems when the sensors, deployed in a distributed manner, may not communicate to an observer at once, and a protocol determines the communication pattern among different sensors. We use the formalism of automata to model the sequence of measurements determined by a protocol and show that the question of observability is decidable for the resulting system. We give upper bounds on the number of measurements required for deciding observability. In addition, we consider the effects of dropouts, which may occur in communicating the measurements across the channel. Again using the formalism of automatons to model certain classes of dropouts combined with the protocol, it is shown that observability is decidable in finite time for measurements sent across using a protocol, and subject to dropouts.
\end{abstract}

\section{INTRODUCTION}

Analysis and design of control systems in the presence of networks between the controllers and the plant has attracted the attention of many researchers in the control community, and there are several survey articles, which summarize the contributions made in this regard in different eras, see, for example, [3], [9], [11]. The importance of fundamental system properties, such as, controllability and observability in dynamical systems, and their role in designing controllers and observers has been well-established. Motivated by these two research directions, we are interested in studying the problems related to observability subject to certain uncertainties which result from operating a system over a digital communication network.

On a system theoretic level, for a system to be observable, the mapping from the initial state to the output needs to be injective. We typically think of the output as some partial information about the state, and in case of communication over a network, this information about the state is further limited, and one looks at alternate mappings, from the state to the measurements received by the receiving node, thereby taking into account the effects of the network. It is thus crucial to study how such properties are affected due to the constraints imposed by the network. In particular, the mappings to be analyzed are determined by the network constraints being considered and modeled. In many practical scenarios, sensors are being equipped with transmitters so that they can send their data to remote computers wirelessly. For large-scale systems, and considering the limited

A. Tanwani is with LAAS - CNRS, University of Toulouse, 7 Ave. Colonel du Roche, Toulouse 31400 France. R. Jungers is a F.R.S.-FNRS Research Associate at ICTEAM Institute, Université catholique de Louvain, Belgium; he is also supported by the French Community of Belgium, the Walloon Region and the Innoviris Foundation. W.P.M.H. Heemels is with Eindhoven University of Technology, The Netherlands. bandwidth of the underlying channel, it is often desirable to transmit data in a sequential manner among these sensors. Using such scenarios as a motivation, we are interested in the problem of analyzing observability when the underlying algorithms or protocols allow individual sensors to communicate independently from the rest, and to consider realistic scenarios, such as measurements being subject to dropouts. As such, in this article, we study observability subject to changes in the measurements which result from introducing both communication protocols and dropouts. In particular, by using the theory of finite state automatons to represent the protocols and dropouts, we investigate whether observability is decidable over all paths generated by the underlying automatons.

In the related literature focused on studying the structural properties in the presence of network uncertainties, several interesting directions have been pursued. From a control viewpoint, the article [6] proposes optimal control algorithms with possible loss in communication at certain time instants. When looking at the constraints arising from communication protocols, the paper [14] studies linear systems with periodic scheduling and provides a sufficient condition to preserve controllability in terms of the eigenvalues of the system and the communication sequence. A generalization of this approach to nonlinear systems can be found in [8]. Another direction is to consider dropouts in communication. The paper [5] considers single-input single-output systems and provides a controllability criterion in the presence of socalled blind periods, or dropouts in communication. More recently, in [7], controllability and observability are studied in the case of dropouts. The authors model the resulting system as a constrained switched system, where in case of a dropout, the authors consider the output to be null, and it switches back to the nominal output in case the transmission is successful.

\section{A. System Class and Problem Overview}

In this article, we are interested in discrete-time linear systems of the form

$$
\begin{aligned}
x(k+1) & =A x(k) \\
y(k) & =C x(k)
\end{aligned}
$$

where $A \in \mathbb{R}^{n \times n}, C \in \mathbb{R}^{\mathrm{d}_{\mathrm{y}} \times n}$, and $x(k) \in \mathbb{R}^{n}$ denotes the state, and $y(k) \in \mathbb{R}^{\mathrm{d}_{\mathrm{y}}}$ denotes the output of the system at time instant $k \in \mathbb{N}:=\{0,1,2, \ldots\}$. For the sake of simplicity, we will assume that the matrix $C$ has no zero rows in it. In the setup of this paper, we consider the scenario where the sensors are deployed in a distributive manner. Instead 
of all the sensors transmitting the output of the system to an observer/state estimator, only a certain selection of these sensors transmit their values across the network at a given time instant. That is, not all the sensors can communicate their data simultaneously across the channel to an observer. Such a situation may arise typically when communicating sensor values across a network, and thus we suppose that a communication protocol determines which sensors must send the data at a given time instant. To differentiate between the output of the plant, and the measurements sent across the network, the measurements received by the receiving node (an observer) at time $k \in \mathbb{N}$ are denoted by $z(k) \in \mathbb{R}^{\mathrm{d}_{k}}$, with $\mathrm{d}_{k} \in \mathbb{N}, \mathrm{d}_{k} \leq \mathrm{d}_{\mathrm{y}}$. Here, $\mathrm{d}_{k}$ denotes the number of sensors that communicate across the network at time instant $k$, as this number may vary with time.

\section{B. Motivation and Examples}

The need for studying observability within the setup of distributed communication can be motivated in several ways. The questions studied in this paper are motivated by the fact that that the observability is not necessarily preserved when the transmission of measurements is affected by some protocol. For instance, consider system (1) with the observable pair $(A, C)$ described by

$$
A=\left[\begin{array}{llll}
0 & 1 & 0 & 0 \\
1 & 0 & 0 & 0 \\
0 & 0 & 0 & 1 \\
0 & 0 & 1 & 0
\end{array}\right] ; \quad C=\left[\begin{array}{llll}
1 & 0 & 0 & 0 \\
0 & 0 & 1 & 0
\end{array}\right] \text {. }
$$

Assume that, a round-robin protocol is used to transmit output data to an observer, which sends $z(2 \ell):=y_{1}(2 \ell)=$ $x_{1}(2 \ell)$, and $z(2 \ell+1):=y_{2}(2 \ell+1)=x_{3}(2 \ell+1)$, for each $\ell \in \mathbb{N}$. It turns out that it is not possible to compute $x(0)$ under this communication protocol. This can be seen by constructing the map from $x(0)$ to the value of $z(k), k \in \mathbb{N}$, and noticing that $A^{2}=I$ for this particular example. Thus, it is of interest to be able to decide whether a given protocol model allows us to reconstruct the state. If so, then what is the maximum number of measurements which guarantee reconstruction of the state. Another related question we ask ourselves is when and how can we construct arbitrarily long sequences of measurements, which do not allow us to reconstruct the state. The proof techniques used in the main results precisely address this kind of questions.

\section{Our Contribution}

Observing these interesting aspects of distributed communication via examples, we propose an automaton-based approach for modeling the protocols. The mapping from the initial state $x(0)$ to the function $z$ varies with time, and by using the properties of the automaton to describe the time-varying map $x(0) \mapsto z$, we address the question whether this mapping is invertible along all paths of an automaton which describes protocols and dropout signals. As we remarked, the mapping $x(0) \mapsto z$ is time-varying under the communication constraints and the overall system can be seen as a switched system, with switching measurement matrices. The approach adopted in [1], [2] provides conservative lower bounds (using tools from number theory) on the time it takes to decide observability, without associating any model to how the measurement matrices change. On the other hand, the approaches adopted in [13], [15] propose observability conditions for a given switching path. This paper, however, takes an intermediate approach between the two, where we provide conditions for deciding observability in finite time over a class of switching paths described by non-deterministic automata. Such partially constrained switched systems have been studied in the context of stability problem recently [4], [10].

\section{Measurement Models With Communication PROTOCOLS}

In this section, the setup of distributed communication is introduced, which is governed by a certain protocol and is affected by dropouts. We make use of a finite state automaton to represent both the protocols and the dropout signals. The underlying properties of the automata are then used to analyze observability related questions.

Definition 1. An automaton is a pair $\mathcal{A}=(M, v) \in$ $\{0,1\}^{N \times N} \times \mathcal{V}^{N}$, where $N$ is the number of nodes (or states), $M \in\{0,1\}^{N \times N}$ is the transition matrix, and $v=$ $\left(\begin{array}{llll}v_{1} & v_{2} & \ldots & v_{N}\end{array}\right)^{\top} \in \mathcal{V}^{N}$ is the vector of node labels, so that $v_{i} \in \mathcal{V}$, for each $i \in\{1, \ldots, N\}$.

Some basic terminology associated to finite state automata, which we use in the paper, now follows: A path $\mathbf{p}$ of length $\ell$ is a sequence of node labels $v_{i_{1}} v_{i_{2}} \ldots v_{i_{\ell}}$, such that $M\left(i_{j}, i_{j+1}\right)=1$, for each $j=1, \ldots, \ell-1$. A path may be of finite or infinite length. A cycle is a path where $v_{i_{1}}=v_{i_{\ell}}$. The restriction of a path $\mathbf{p}$, described as $\mathbf{p}_{[1: k]}=$ $v_{i_{1}} v_{i_{2}} \ldots v_{i_{k}}, k \leq \ell$, is also a path. If we have two paths $\mathbf{a}$ and $\mathbf{b}$ given by $v_{1}^{a} v_{2}^{a} \ldots v_{\ell^{a}}^{a}$, and $v_{1}^{b} v_{2}^{b} \ldots v_{\ell^{b}}^{b}$, then we can define their concatenation to construct a feasible path if $v_{\ell^{a}}^{a}=v_{1}^{b}$, and the resulting concatenated path of length $\ell^{a}+\ell^{b}-1$ is $\mathbf{a b}:=v_{1}^{a} v_{2}^{a} \ldots v_{\ell^{a}}^{a} v_{2}^{b} \ldots v_{\ell^{b}}^{b}$. We use the notation $\mathbf{a}^{(m)}$ to represent a path, where a is concatenated $m$ times with itself. Clearly, this applies to the case where $\mathbf{a}$ is a cycle. We now use these notions to describe the communication model for the sensors.

\section{A. Protocol Description}

In our setup of distributed communication, a protocol makes a selection, at each time instant $k \in \mathbb{N}$, to send the values measured from some subset of the sensors. To formally define the class of protocols in this paper, consider the $P$ subsets $\mathcal{S}_{1}, \ldots, \mathcal{S}_{P}$ with distinct elements, so that $\mathcal{S}_{j} \subset\left\{1,2, \ldots, \mathrm{d}_{\mathrm{y}}\right\}$ and $\operatorname{card}\left(\mathcal{S}_{j}\right)=: \mathrm{d}_{j}, j=1, \ldots, P$. That is, we choose $P$ elements from the power set of $\left\{1, \ldots, \mathrm{d}_{\mathrm{y}}\right\}$. We do not require the subsets $\mathcal{S}_{j}, j=1,2, \ldots, P$, to be distinct. Each $\mathcal{S}_{j}$ indicates the set of indices of the sensors that a protocol may select at a given time instant. A protocol, as considered in this paper, specifies a scheduling signal $\mathcal{S}: \mathbb{N} \rightarrow\left\{\mathcal{S}_{1}, \ldots, \mathcal{S}_{P}\right\}$, so that the measurement $z(k)$ sent across the channel at time instant $k \in \mathbb{N}$ is the vector composed of $y_{i}(k)$ with $i \in \mathcal{S}(k)$. 
Here, we emphasize that a protocol may allow different scheduling signals $\mathcal{S}$ due to nondeterministic transition rules for switching among sensor values. This phenomenon is appropriately captured by using an automaton to model all the possibilities described by a protocol.

Definition 2. A protocol automaton is a pair $\mathcal{A}_{p}=$ $\left(M_{p}, s\right) \in\{0,1\}^{P \times P} \times\left\{\mathcal{S}_{1}, \ldots, \mathcal{S}_{P}\right\}^{P}$, where the vector $s=\left(\begin{array}{llll}s_{1} & s_{2} & \ldots & s_{P}\end{array}\right)^{\top} \in\left\{\mathcal{S}_{1}, \ldots, \mathcal{S}_{P}\right\}^{P}$. A scheduling signal $\mathcal{S}: \mathbb{N} \rightarrow\left\{\mathcal{S}_{1}, \ldots, \mathcal{S}_{P}\right\}$ is considered admissible with respect to $\mathcal{A}_{p}$ if there exists $w_{p}: \mathbb{N} \rightarrow\{1, \ldots, P\}$ such that $M_{p}\left(w_{p}(k), w_{p}(k+1)\right)=1$ and $\mathcal{S}(k)=\mathcal{S}_{w_{p}(k)}$, for all $k \in \mathbb{N}$.

We introduce the matrices $S_{j} \in \mathbb{R}^{\mathrm{d}_{j} \times n}$ associated to the node $\mathcal{S}_{j}$, whose rows are exactly the rows of matrix $C$ represented by $\mathcal{S}_{j}$. In other words, $i \in \mathcal{S}_{j}$ if and only if the $i$-th row of $C$ is a row of $S_{j}$. With an admissible scheduling signal $\mathcal{S}$, we associate a measurement signal $R_{\mathcal{S}}: \mathbb{N} \rightarrow$ $\left\{S_{1}, \ldots, S_{p}\right\}$, such that $R_{\mathcal{S}}(k)=S_{i_{k}}$ if $\mathcal{S}(k)=\mathcal{S}_{i_{k}} k \in \mathbb{N}$.

For a path $\mathbf{p}=\mathcal{S}_{i_{1}} \mathcal{S}_{i_{2}} \cdots \mathcal{S}_{i_{\ell}}$, of the automaton $\mathcal{A}_{p}$, we define its rank, denoted $\operatorname{rank}(\mathbf{p})$, as ${ }^{1}$

$$
\operatorname{rank}(\mathbf{p})=\operatorname{rank}\left(\operatorname{col}\left(S_{i_{1}}, S_{i_{2}} A, \ldots, S_{i_{\ell}} A^{\ell-1}\right)\right),
$$

where the matrix on the right-hand side is understood to have $n$ columns, and the number of rows depends on the path $\mathbf{p}$.

Example 1 (Round-robin protocol with $y \in \mathbb{R}^{\mathrm{d}_{\mathrm{y}}}$ ). Consider the case when there are $\mathrm{d}_{\mathrm{y}}$ sensors and $y_{i}=x_{i}, i=$ $1, \ldots, \mathrm{d}_{\mathrm{y}}$, and $x \in \mathbb{R}^{n}$, with $n \geq \mathrm{d}_{\mathrm{y}}$. We choose $P=\mathrm{d}_{\mathrm{y}}$, and $\mathcal{S}_{i}=\{i\}$, that is, each sensor sends the value of one of the state components. A round-robin protocol, that chooses,

$$
z\left(\mathrm{~d}_{\mathrm{y}} \ell+j\right)=y_{j}\left(\mathrm{~d}_{\mathrm{y}} \ell+j\right), \quad j=1, \ldots, \mathrm{d}_{\mathrm{y}},
$$

falls in this framework. The corresponding automaton is described by choosing $s_{j}=\mathcal{S}_{j}, j=1, \ldots, P$, and the nonzero entries of $M_{p}$ are given by $M_{p}(i, i+1)=1$, for $i=1, \ldots, P-1$. The resulting automaton is a simple cycle as shown in Figure 1, where we draw an edge from $\mathcal{S}_{i}$ to $\mathcal{S}_{j}$ only if $M_{p}(i, j)=1$.

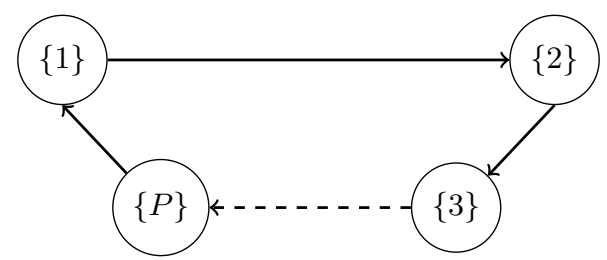

Fig. 1: An automaton for round-robin protocols.

Example 2 (Protocol with limited tokens). Consider the case where $y \in \mathbb{R}^{2}$, and the protocol under consideration is such that, at each time instant, it chooses to send $y_{i}, i=1,2$, but the constraint is that it can not send the value from the same sensor more than two times consecutively. That is, neither $y_{1}$, nor $y_{2}$, can be sent more than twice in a row. This protocol

\footnotetext{
${ }^{1}$ Given the matrices $M_{1}, \ldots, M_{k}$ with same number of columns, we use the notation $\operatorname{col}\left(M_{1}, \ldots, M_{k}\right):=\left[\begin{array}{lll}M_{1}^{\top} & \ldots & M_{k}^{\top}\end{array}\right]^{\top}$.
}

rule introduces non-determinism which can be modeled by choosing $P=4$, and $\mathcal{S}_{1}=\mathcal{S}_{2}=\{1\}$, and $\mathcal{S}_{3}=\mathcal{S}_{4}=\{2\}$, where the corresponding automaton is given in Fig. 2.

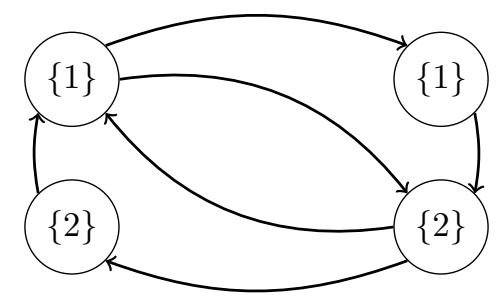

Fig. 2: A protocol automaton with limited tokens assigned to each sensor.

\section{B. Modeling Dropouts}

So far, we considered the fact that the presence of a communication channel does not allow all the sensors to communicate at once, and different sensors transmit their measurements at different times according to the scheduling constraints governed by a protocol. In addition, we also want to study the case where a transmitted packet drops before reaching the destination. We again use a finite state automaton to model certain classes of dropouts and will subsequently analyze observability for such cases.

Definition 3. A dropout automaton is an automaton $\mathcal{A}_{d}=\left(M_{d}, v\right) \in\{0,1\}^{N \times N} \times\{0,1\}^{N}$, where $v=$ $\left(\begin{array}{llll}v_{1} & v_{2} & \ldots & v_{N}\end{array}\right)^{\top} \in\{0,1\}^{N}$ is such that $v_{i}=0$ represents a dropout in communication, and $v_{i}=1$ denotes a node with successful transmission. A data loss signal $\sigma: \mathbb{N} \rightarrow\{0,1\}$ is said to be admissible with respect to a dropout automaton $\mathcal{A}_{d}=\left(M_{d}, v\right)$ with $N$ nodes, if there exists a sequence of states $w_{d}: \mathbb{N} \rightarrow\{1,2, \ldots, N\}$ such that for all $k \in \mathbb{N}$ it holds that $M\left(w_{d}(k), w_{d}(k+1)\right)=1$ and $\sigma(k)=v_{w_{d}(k)}$.

One can check that, several dropout models can be encoded using the formalism of automatons and we refer the reader to [7] for several examples.

\section{Measurement Model and Observability Notions}

For the communication setup considered in this paper, the effect of the channel on the measurements is described by the protocol automaton $\mathcal{A}_{p}$ and the dropout automaton $\mathcal{A}_{d}$. For a scheduling signal $\mathcal{S}$ admissible with respect to $\mathcal{A}_{p}$, and a data-loss signal $\sigma$ admissible with respect to $\mathcal{A}_{d}$, we can now consider the system

$$
\begin{aligned}
x(k+1) & =A x(k) \\
z(k) & =\sigma(k) R_{\mathcal{S}}(k) x(k),
\end{aligned}
$$

where we recall that $R_{\mathcal{S}}$ is the matrix-valued measurement signal associated to $\mathcal{S}$. The observability question of interest is formulated as recovering the state $x$ using the networkaffected measurements $z$, which are described by equation (2).

Definition 4. The system defined by the quadruple $\left(A, C, \mathcal{A}_{p}, \mathcal{A}_{d}\right)$ is called observable if for each scheduling 
signal $\mathcal{S}$ and each data-loss signal $\sigma$ that are admissible with respect to $\mathcal{A}_{p}$ and $\mathcal{A}_{d}$, respectively, the implication

$$
z \equiv 0 \quad \Rightarrow \quad x_{0}=0
$$

holds, where $z$ is obtained from (2) with the initial condition $x(0)=x_{0}$.

Because of the linearity of system under consideration, the above implication is equivalent to

$$
z_{1} \equiv z_{2} \quad \Rightarrow \quad x_{1}(0)=x_{2}(0),
$$

where $z_{1}$ and $z_{2}$ are the measurements sent across the channel when system (1) is initialized with $x_{1}(0)$ and $x_{2}(0)$, respectively. Checking observability boils down to checking whether the matrix

$$
\operatorname{col}\left(\sigma(1) R_{\mathcal{S}}(1), \sigma(2) R_{\mathcal{S}}(2), \ldots\right)
$$

has rank $n$ for all possible signals $\mathcal{S}, \sigma$ generated by the automata $\mathcal{A}_{p}$ and $\mathcal{A}_{d}$, respectively, which is a question addressed in next two sections.

\section{OBSERVABILITY Without DRopouts}

Observability of the system using the network-affected measurements introduced in the previous section is now investigated. For the sake of clarity, we first present our results in this section without the dropouts and then prove similar results including dropouts in Section IV. When talking about system without dropouts, we say that the system $\left(A, C, \mathcal{A}_{p}\right)$ is observable if (3) holds for each $\mathcal{S}$ admissible to $\mathcal{A}_{p}$ while fixing $\sigma \equiv 1$ in (2). Our strategy to address this question is to first look at the case of simple cyclic automata $\mathcal{A}_{p}$, which we present in Section III-A. We then build on this result to show that observability over all paths in a protocol automaton $\mathcal{A}_{p}$ can be checked by looking at the rank of finitely many matrices.

\section{A. Observability for Simple Cyclic Automatons}

To present conditions for observability, we introduce some notation. For a given pair $(A, C)$, with $A \in \mathbb{R}^{n \times n}$, we introduce the matrix $\mathcal{O}_{(A, C)}$ to be

$$
\mathcal{O}_{(A, C)}:=\operatorname{col}\left(C, C A, \cdots, C A^{n-1}\right) .
$$

The pair $(A, C)$ is called observable in the classical sense if $\mathcal{O}_{(A, C)}$ has full column rank.

We first consider the case of cyclic protocol automata without dropouts. For this case, we introduce the notation:

$$
\widetilde{C}:=\operatorname{diag}\left(S_{1}, S_{2}, \ldots, S_{P}\right)
$$

and let $\widetilde{A}:=\Pi_{P}^{\top} \otimes A$, with $\Pi_{P}$ being the canonical cyclic permutation matrix on $P$ elements, and $\otimes$ denotes the Kronecker product of matrices.

Proposition 1. The system defined by the triplet $\left(A, C, \mathcal{A}_{p}\right)$ with cyclic protocol automaton $\mathcal{A}_{p}$ is observable if and only if

$$
\operatorname{rank} \mathcal{O}_{(\widetilde{A}, \widetilde{C})}=n P
$$

Proof. Assume that (4) holds. Let us consider the measurements of system (2) over the interval $[0, P-1]$, denoted by $z_{[0, P-1]}$, that is, $z_{[0, P-1]}:=\operatorname{col}(z(0), z(1), \cdots, z(P-1))=$ $\bar{R} x(0)$, where $\bar{R}:=\operatorname{col}\left(R(1), R(2) A, \ldots, R(P) A^{P-1}\right)$. This leads to

$$
\left(\begin{array}{c}
z_{[0, P-1]} \\
z_{[P, 2 P-1]} \\
\cdots \\
z_{[(n-1) P, n P-1]}
\end{array}\right)=\left[\begin{array}{c}
\bar{R} \\
\bar{R} A^{P} \\
\cdots \\
\bar{R}\left(A^{P}\right)^{n-1}
\end{array}\right] x(0)
$$

On the other hand, the matrix $\mathcal{O}_{(\widetilde{A}, \widetilde{C})}$ has $n P$ columns and hence each set of $n$ columns is linearly independent. In particular, for each $k=0, \cdots, P-1$, the submatrix formed by taking the columns $k n+1, k n+2, \ldots, k n+n$ has rank $n$. But, by construction, one of these submatrices corresponds to the matrix appearing in the right-hand side of (5). This shows that (2) is observable if (4) holds.

We prove the other implication by contradiction. Assume that the matrix $\mathcal{O}_{(\widetilde{A}, \widetilde{C})}$ has rank less than $n P$. There exists $\widetilde{x}$ such that $\mathcal{O}_{(\widetilde{A}, \widetilde{C})} \widetilde{x}=0$. The structure of $\mathcal{O}_{(\widetilde{A}, \widetilde{C})}$ is such that, for some $k=0, \cdots, P-1$, the subvector $\widetilde{x}_{[k n+1: k n+n]} \neq 0$ is in the null space of the matrix formed by the columns $k n+1, k n+2, \ldots, k n+n$. Hence, it is possible to generate a measurement signal $R$ compatible with $\mathcal{A}_{p}$ such that the right-hand side of (5) equals zero by taking $x(0)=$ $\widetilde{x}_{[k n+1: k n+n]}$. This makes (2) unobservable, which proves the necessity of (4).

The lifting technique adopted in Proposition 1 allows us to formulate the observability question for the system with switching measurement matrices in terms of the observability of a linear time-invariant system. This result also contains the idea needed to solve the case of more general protocols.

\section{B. Observability with Arbitrary Protocol Automatons}

We next move to analyzing observability under protocols, where the representative automaton may include nondeterministic transitions. The statement of Proposition 1 reveals that in case of a simple cycle, deciding whether a given path is observable is relatively straightforward and can be done by looking at a string of length $n P$ generated by the automaton $\mathcal{A}_{p}$. For more complex automata, we can build on this result to address the question whether observability can be decided in finite time for all possible strings that can be generated by $\mathcal{A}_{p}$. The answer to this question is provided in the affirmative in Theorem 1.

The basic idea of the proof is the following observation: if each node in the automaton $\mathcal{A}_{p}$ corresponds to a nontrivial measurement, then a path of length 1 ensures that the corresponding rank of the observability matrix is at least 1. Next, if we consider a path of length $P+1$, with $P$ being the number of nodes, it follows from the Pigeon Hole principle that at least one node must appear twice, and hence there is at least one cycle in that path. If there is a path containing this cycle such that the rank of the observability matrix corresponding to that path is again 1 , it can be shown that going around that cycle does not increase the rank of the observability matrix. If we now construct paths of arbitrary 
length by repeating this cycle (by the so-called pumping lemma in Automata theory) such paths are still feasible but the rank of the observability matrix does not increase. In other words, just by checking if the rank of the observability matrix is less than 2 in a cycle of length less than $P+1$, we can construct paths of arbitrary length where the rank of the observability matrix stays less than 2 .

Extending this line of thought, we can find a positive integer $\eta^{*}$ such that it suffices to check if the observability matrices corresponding to all paths of length $\eta^{*}$ have rank $n$. If not, then there is a cycle (not necessarily simple) in the path, which can be used to construct paths of arbitrary length resulting in unobservability. With this motivation let us introduce the following sequence of numbers:

$$
\begin{aligned}
& \eta(1)=1 \\
& \eta(\ell)=\eta(\ell-1)+L(\eta(\ell-1)), \quad 2 \leq \ell \leq n,
\end{aligned}
$$

where $L(\eta)$ denotes the number of paths of length $\eta$ which can be generated by $\mathcal{A}_{p}$.

Theorem 1. The system $\left(A, C, \mathcal{A}_{p}\right)$ is observable if and only if every path of $\mathcal{A}_{p}$, with length at least $\eta(n)$, has rank $n$.

Remark 1. For simple cyclic automatons with $P$ nodes, it is readily checked that $L(k)=P$ for all $k \in \mathbb{N}_{\geq 1}$, and so $\eta(n)=n P+1$, which is very close to the number given in Proposition 1. For more complex graphs, the number $\eta(n)$ may be very large and only provides a conservative estimate on the length of the paths for which we need to check the rank. Getting tighter lower bounds for lengths of paths for which we need to check observability for particular classes of automatons is an interesting question that requires further investigation.

The following lemma is used in the proof of Theorem 1.

Lemma 1. If $\mathbf{s}$ denotes a path in an automaton $\mathcal{A}_{p}$, and $\mathbf{s r s}$ is a path such that

$$
\operatorname{rank}(\mathbf{s r s})=\operatorname{rank}(\mathbf{s})
$$

then for any arbitrary integer $m \geq 1$, the path $\mathbf{s}(\mathbf{r s})^{(m)}$ has the same rank as the path $\mathbf{s}$.

Proof. Let $\mathbf{s}$ be a path of length $s_{\ell}$ and $\mathbf{r}$ be a path of length $r_{\ell}$. The equality in (6) says that

$$
\operatorname{rank}\left[\begin{array}{c}
O_{\mathbf{s}} \\
O_{\mathbf{r}} A^{s_{\ell}} \\
O_{\mathbf{s}} A^{s_{\ell}+r_{\ell}}
\end{array}\right]=\operatorname{rank} O_{\mathbf{s}}
$$

where $O_{\mathbf{s}}:=\operatorname{col}\left(C_{s_{1}}, C_{s_{2}} A, \cdots, C_{s_{\ell}} A^{s_{\ell}-1}\right)$, and $O_{\mathbf{r}}:=$ $\operatorname{col}\left(C_{r_{1}} C_{r_{2}} A \cdots C_{r_{\ell}} A^{r_{\ell}-1}\right)$. Let us now look at the matrix associated with the path $\mathbf{s}(\mathbf{r s})^{(2)}$. To establish that the rank of the path $\mathbf{s}(\mathbf{r s})^{(2)}$ is the same as that of $\mathbf{s}$, we show that

$$
\operatorname{rank}\left[\begin{array}{c}
O_{\mathbf{s}} \\
O_{\mathbf{r}} A^{s_{\ell}} \\
O_{\mathbf{s}} A^{s_{\ell}+r_{\ell}} \\
O_{\mathbf{r}} A^{2 s_{\ell}+r_{\ell}} \\
O_{\mathbf{s}} A^{2 s_{\ell}+2 r_{\ell}}
\end{array}\right]=\operatorname{rank} O_{\mathbf{s}}
$$

from where the statement of the lemma follows by induction. To prove the foregoing equality, the lower portion of the matrix on left-hand side is written as

$$
\left[\begin{array}{c}
O_{\mathbf{s}} A^{s_{\ell}+r_{\ell}} \\
O_{\mathbf{r}} A^{2 s_{\ell}+r_{\ell}} \\
O_{\mathbf{s}} A^{2 s_{\ell}+2 r_{\ell}}
\end{array}\right]=\left[\begin{array}{c}
O_{\mathbf{s}} \\
O_{\mathbf{r}} A^{s_{\ell}} \\
O_{\mathbf{s}} A^{s_{\ell}+r_{\ell}}
\end{array}\right] A^{s_{\ell}+r_{\ell}}
$$

and hence

range $\left[\begin{array}{c}O_{\mathbf{s}} A^{s_{\ell}+r_{\ell}} \\ O_{\mathbf{r}} A^{2 s_{\ell}+r_{\ell}} \\ O_{\mathbf{s}} A^{2 s_{\ell}+2 r_{\ell}}\end{array}\right]^{\top}=\left(A^{s_{\ell}+r_{\ell}}\right)^{\top}$ range $\left[\begin{array}{c}O_{\mathbf{s}} \\ O_{\mathbf{r}} A^{s_{\ell}} \\ O_{\mathbf{s}} A^{s_{\ell}+r_{\ell}}\end{array}\right]^{\top}$.

It follows from (7) that

$$
\operatorname{range}\left[\begin{array}{c}
O_{\mathbf{s}} \\
O_{\mathbf{r}} A^{s_{\ell}} \\
O_{\mathbf{s}} A^{s_{\ell}+r_{\ell}}
\end{array}\right]^{\top}=\operatorname{range} O_{\mathbf{s}}^{\top},
$$

which gives

$$
\text { range }\left[\begin{array}{c}
O_{\mathbf{s}} A^{s_{\ell}+r_{\ell}} \\
O_{\mathbf{r}} A^{2 s_{\ell}+r_{\ell}} \\
O_{\mathbf{s}} A^{2 s_{\ell}+2 r_{\ell}}
\end{array}\right]^{\top}=\left(A^{s_{\ell}+r_{\ell}}\right)^{\top} \text { range } O_{\mathbf{s}}^{\top} .
$$

Since the matrix on the right-hand side of (10) is contained inside the matrix appearing on the left-hand side of (9), the desired relation in (8) thus follows.

Proof of Theorem 1. Consider a path $\mathbf{p}$ of length greater than or equal to $\eta(n)$ generated by the automaton $\mathcal{A}_{p}$. For any integer $\ell \in\{1, \ldots, n\}$, we show that either

$$
\operatorname{rank}\left(\mathbf{p}_{[1: \eta(\ell)]}\right) \geq \ell,
$$

or there exists a path $\mathbf{q}$ of arbitrary length such that

$$
\operatorname{rank}(\widetilde{\mathbf{p q}})<n
$$

where $\widetilde{\mathbf{p}}$ is some restriction of the path $\mathbf{p}$. Let us start by looking at $\mathbf{p}_{[1: \eta(2)]}$ whose length ${ }^{2}$ is $\eta(2)$. There exist $1 \leq i<j \leq \eta(2)$ such that $\mathbf{p}_{[i]}=\mathbf{p}_{[j]}$. If $\operatorname{rank}\left(\mathbf{p}_{[1: \eta(2)]}\right)<$ 2 , then by invoking Lemma 1 (with $\mathbf{s}=\mathbf{p}_{[i]}$, and $\mathbf{r}=$ $\left.\mathbf{p}_{[i: j]}\right)$, and letting $\mathbf{q}=\left(\mathbf{p}_{[i: j]}\right)^{(m)}, \widetilde{\mathbf{p}}=\mathbf{p}_{[i]}$, we see that $\operatorname{rank}(\widetilde{\mathbf{p}} \mathbf{q})=1$. Thus, we continue with rank of $\mathbf{p}_{[1: \eta(2)]}$ at least rank 2.

For the sake of induction, assume that $\mathbf{p}_{[1: \eta(\ell)]}$ has at least rank $\ell, 2 \leq \ell<n$, and consider the path $\mathbf{p}_{[1: \eta(\ell+1)]}$. Assume that $\mathbf{p}_{[1: \eta(\ell+1)]}$ has rank $\ell$. By the Pigeon Hole principle, there exists $1 \leq i<j<L(\eta(\ell))$ such that $\mathbf{p}_{[i: i+\eta(\ell)]}=$ $\mathbf{p}_{[j: j+\eta(\ell)]}$ and $\operatorname{rank} \mathbf{p}_{[i: j+\eta(\ell)]}=\operatorname{rank} \mathbf{p}_{[i: i+\eta(\ell)]}$. Applying Lemma 1 with $\mathbf{s}=\mathbf{p}_{[i: i+\eta(\ell)]}, \mathbf{r}=\mathbf{p}_{[i+\eta(\ell): j]}$, we can then let $\widetilde{\mathbf{p}}=\mathbf{p}_{[1: i+\eta(\ell)]}$, and

$$
\mathbf{q}=\mathbf{p}_{[i+\eta(\ell): j+\eta(\ell)]}^{(m)}
$$

to deduce that $\operatorname{rank}(\widetilde{\mathbf{p q}})=\ell$. This means we have either constructed a path of arbitrary length with rank equal to $\ell<n$, or the path $\mathbf{p}_{[1: \eta(\ell+1)]}$ must at least have rank $\ell+1$. Proceeding inductively and arriving at $\ell=n-1$ establish the proof of the desired result.

\footnotetext{
${ }^{2}$ Note that $\eta(2)=1+L(1)$, and the total number of paths of length 1 , that is $L(1)$, is just the number of nodes.
} 


\section{OBSERVABILITY WITH DROPOUTS}

We now state our main result for observability of system (2) with $R$ and $\sigma$ generated by any given automaton $\mathcal{A}_{p}$ and $\mathcal{A}_{d}$. We aim to seek the same kind of results as in the previous section, that is, whether the problem is decidable or not. The basic idea behind our approach is to first construct a single automaton from $\mathcal{A}_{p}$ and $\mathcal{A}_{d}$, and then study the observability question for this resulting automaton in the same manner as done in Theorem 1.

\section{A. Construction of Augmented Automaton $\widetilde{\mathcal{A}}$}

The main algebraic ingredient in this first step resides in taking the Kronecker product of the matrices from the two automata, pairwise. Toward this end, we represent the automata via the adjacency matrices corresponding to their different nodes. That is, $\mathcal{A}_{d}$ contains two matrices $M_{0}, M_{1} \in$ $\{0,1\}^{N \times N}$, where $N$ is the number of nodes in the dropout automaton, and the $(i, j)$-entry of $M_{0}$ (resp. $M_{1}$ ) is equal to 1 if and only if, in $\mathcal{A}_{d}$, there is an edge from node $i$ to node $j$ and the label of node $j$ is equal to zero (resp. one). Similarly for $\mathcal{A}_{p}$, which is an automaton on $P$ nodes: the matrix $M_{\mathcal{S}_{i}} \in\{0,1\}^{P \times P}$ describes the edges of $\mathcal{A}_{p}$ that point to a node with label $\mathcal{S}_{i}$.

Now, we describe our new automaton $\widetilde{\mathcal{A}}$ via its matrices. The automaton has $N \cdot P$ nodes, and the matrices $\widetilde{M}_{S_{i}}$ are given by the Kronecker product

$$
\widetilde{M}_{\mathcal{S}_{i}}:=M_{\mathcal{S}_{i}} \otimes M_{1} \in\{0,1\}^{N P \times N P},
$$

while the matrix $\widetilde{M}_{0}$ is given by

$$
\widetilde{M}_{0}:=M_{p} \otimes M_{0} \in\{0,1\}^{N P \times N P},
$$

where $M_{p}$ is the adjacency matrix describing all the edges of $\mathcal{A}_{p}$. One can verify that this construction provides the automaton $\tilde{\mathcal{A}}$ described by the pair $(\widetilde{M}, \widetilde{v})$, where $\widetilde{M}:=\widetilde{M}_{0}+$ $\sum_{i=1}^{P} \widetilde{M}_{\mathcal{S}_{i}}$ is the transition matrix, and $\widetilde{v} \in\left\{\mathcal{S}_{1}, \ldots, \mathcal{S}_{P}\right\}^{P} \times$ $\{0,1\}^{N}$ is the vector of node labels. This automaton $\widetilde{\mathcal{A}}$ indeed generates all the signals that are compliant, both with the protocol and the dropout automata. That is, for example, an observability matrix

$$
\operatorname{col}\left(0, S_{i_{4}}, 0,0, S_{i_{1}}\right)
$$

is admissible if and only if the corresponding product $\widetilde{M}_{0} \widetilde{M}_{\mathcal{S}_{i_{4}}} \widetilde{M}_{0} \widetilde{M}_{0} \widetilde{M}_{\mathcal{S}_{i_{1}}}$ is nonzero.

\section{B. Decidability Result}

As done before, we associate a sequence of numbers with this augmented automaton $\widetilde{\mathcal{A}}$ to decide observability. We let $\mu$ be a sequence of numbers defined as:

$$
\begin{aligned}
& \mu(1)=N+1, \\
& \mu(\ell)=\mu(\ell-1)+L(\mu(\ell-1)),
\end{aligned}
$$

where $L(\mu)$ denotes the total number paths of length $\mu$ that can be generated by the automaton $\widetilde{\mathcal{A}}$.

Theorem 2. The system described by the quadruple $\left(A, C, \mathcal{A}_{p}, \mathcal{A}_{d}\right)$ is observable if and only if every path of the automaton $\widetilde{\mathcal{A}}$, with length at least $\mu(n)$, has rank $n$.
The proof of Theorem 2 essentially follows the same arguments as Theorem 1. One notable difference arises due to the construction of the sequence $\mu$ for the automaton $\widetilde{\mathcal{A}}$, compared to the sequence $\eta$ used for $\mathcal{A}_{p}$. Since the automaton $\widetilde{\mathcal{A}}$ contains possible $N$ nodes with 0 matrix associated to them, one needs to look at paths of length $N+1$ to obtain an observability matrix of rank 1 along such paths. The remainder of the proof then follows in an identical manner.

\section{CONCLUSiOns}

One can build on this work to obtain more constructive algorithms for decidability and reduce the bound on the number of measurements that are required for checking observability with given automata. The question of reconstructing the state in optimal time for control purposes is also of practical relevance.

\section{REFERENCES}

[1] M. Babaali and M. Egerstedt. Pathwise observability and controllability are decidable. In Proc. 42nd IEEE Conf. on Decision and Control, 5771-5776, 2003.

[2] M. Babaali and M. Egerstedt. Observability of switched linear systems. In R. Alur and G.J. Pappas, editors, Hyb. Sys. Computation \& Control, Lecture Notes in Computer Science, pages 48-63. Springer, BerlinHeidelberg, 2004.

[3] A. Bemporad, M. Heemels, and M. Johansson, editors. Networked Control Systems, volume 46 of Lecture Notes in Control and Information Sciences. Springer-Verlag, Berlin, 2010.

[4] X. Dai. A Gelfand-type spectral radius formula and stability of linear constrained switching systems. Linear Algebra and its Applications, 436:1099-1113, 2012.

[5] A. D'Innocenzo, M. Di Benedetto, and E. Serra. Fault tolerant control of multi-hop control networks. IEEE Transactions on Automatic Control, 58(6):1377-1389, 2013.

[6] O. Imer, S. Yuksel, and T. Basar. Optimal control of LTI systems over unreliable communication networks. Automatica, 42(9):14291439, 2006.

[7] R. Jungers, A. Kundu, and W.P.M.H. Heemels. Observability and controllability analysis of linear systems subject to data losses. to appear in IEEE Transactions on Automatic Control; Online: https://arxiv.org/abs/1609.05840.

[8] M. Ljesnjanin, D.E. Quevedo, and D. Nesic. Controllability of discrete-time networked control systems with try once discard protocol. In Proc.19th IFAC World Congress, pages 3758-3763, 2014.

[9] M. Pajic, S. Sundaram, G.J. Pappas, and R. Mangharam. The wireless control network: A new approach for control over networks. IEEE Transactions on Automatic Control, 56:2305-2318, 2011.

[10] M. Philippe, R. Essick, G. Dullerud, and R.M. Jungers. Stability of discrete-time switching systems with constrained switching sequences. Automatica, 72:242-250, 2015

[11] L. Schenato, M. Francesschetti, K. Poolla, and S.S. Sastry. Foundations of control and estimation over lossy networks. Proceedings of the IEEE, 95:163-187, 2007.

[12] T. Skolem. Ein verfahren zur behandlung gewisser exponentialer gleichungen und diophantischer gleichungen. Mathematica Scandinavica, 163-188, 1934.

[13] Z. Sun and S.S. Ge. Switched Linear Systems: Control and Design. Springer-Verlag London, 2005.

[14] T. Suzuki, M. Kono, N. Takashi, and O. Sato. Controllability and stabilizability a networked control system with periodic communication constraints. System \& Control Letters, 60(12):977 - 984, 2014.

[15] A. Tanwani, H. Shim, and D. Liberzon. Observability for switched linear systems: characterization and observer design. IEEE Trans. Automatic Control, 58(4):891 - 904, 2013.

[16] E.P. van Horssen, A.R.B. Behrouzin, D. Goswami, D. Antunes, T. Basten, and W.P.M.H. Heemels. Performance analysis and controller improvement for linear systems with $(m, k)$-firm data losses. In Proc. of the European Control Conference, 2016. 